\title{
Drug Binding, Order-Order and Order-Disorder Transitions in DNA Triple Helices
}

\author{
Seema Srivastava, Vishwambhar Dayal Gupta, ${ }^{\dagger}$ Poonam Tandon, ${ }^{*}$ \\ Shyam SingH, and Seturam Bandhachar KATTI \\ Divison of Biopolymers, Central Drug Research Institute, Lucknow 226001 , India ${ }^{\dagger \dagger}$ \\ * Physics Department, Lucknow University, Lucknow 226 007, India
}

(Received June 24, 1997)

\begin{abstract}
Zimm and Bragg theory for helix to coil transition has been suitably amended and applied to explain the two step transition in DNA triplex. The experimental measurements reported by G. E. Plum et al. and Y. W. Park et al. have been interpreted. The order-order and order-disorder transitions associated with the melting of DNA triple helix, with and without netropsin binding, have been characterized by the nucleation parameter, enthalpy change, sharpness of transition, and heat capacity. The destabilization of the triplex and stabilization of duplex on netropsin binding are reflected in these characteristic parameters.
\end{abstract}

KEY WORDS DNA Triplex / Phase Transition / Sharpness of Transition / Minor Groove Binding /

Nucleic acid complexes containing DNA and RNA type segments have been of considerable value in the elucidation of the structures and physical properties of nucleic acids. In this context ${ }^{1-15}$ various structure like Poly (A·U), Poly $(U \cdot A \cdot U)$, Poly $(d A \cdot d T)$, Poly $(d T \cdot d A \cdot$ $\mathrm{dT}$ ), etc. have been studied. It is well established that DNA triple helices can be formed by the association of deoxyribohomopyrimidine polymers with complementary double helical homopurine-homopyrimidine complexes. The third homopyrimidine strand binds via Hoogsteen base pairing to the purine base in the major groove of the Watson-Crick double helix forming base triplets and lying parallel to the Watson-Crick homopurine strand (Figure 1). Triple helices consisting of homopurine strand as the third strand also have been observed. $^{16}$

Interest in triplexes has been generated by the enzymic, chemical, and physical properties of purine-pyridimine inserts in plasmids. A mirror repeat in the purine-pyrimidine sequence is essential for intra molecular triplex formation because of the specificity for the interactions between the bases in the triads. It is this mirror symmetry of the third strand which is probably responsible for its helical nature. Such biomolecular triplexes have been observed in the structure of $\mathrm{H} \mathrm{DNA}^{17}$ which contains both single stranded and triple stranded regions.

The biological function of triple stranded DNA is not yet clear. Nevertheless, there is growing conviction that this unusual structure may play an important role in gene expression, enhanced sensitivity to chemical cleavage and single-strand specific endonucleases. The structure can be modified to alter enzyme cutting by selectively blocking enzyme binding sites in the major groove. Triplex formation is used as a powerful tool for sequence specific recognition of DNA. In some cases the formation of triplex is used in recognizing a mixed double stranded DNA sequence.

These DNA triple helices undergo simple reversible

\footnotetext{
${ }^{\dagger}$ To whom all correspondence should be addressed
}

†+ Communication No. 5724. helix to random coil transition under different environmental conditions including when bound to drugs like netropsin and dystamycin.4,9-11,18-20 Recently, Plum et $a l .{ }^{9}$ and Park et $a l .{ }^{10}$ reported spectroscopic and calorimetric studies on the DNA triplexes made of $\mathrm{Y} 15 \cdot \mathrm{U} 21 \cdot \mathrm{Y} 21$. Here $\mathrm{U}$ and $\mathrm{Y}$ stand for purine and pyrimidine strands, the subscripts stand for the number of bases ( 30 and 42 base pairs). In their case helix to coil transition has been characterized by a cooperative absorbance change as a function of temperature. The melting of triplex occurs in two steps. The low temperature step is $\mathrm{pH}$ dependent and assigned to the thermal expulsion of the third strand. The high temperature transition is $\mathrm{pH}$ independent and assigned to the thermal disruption of the duplex to form the single

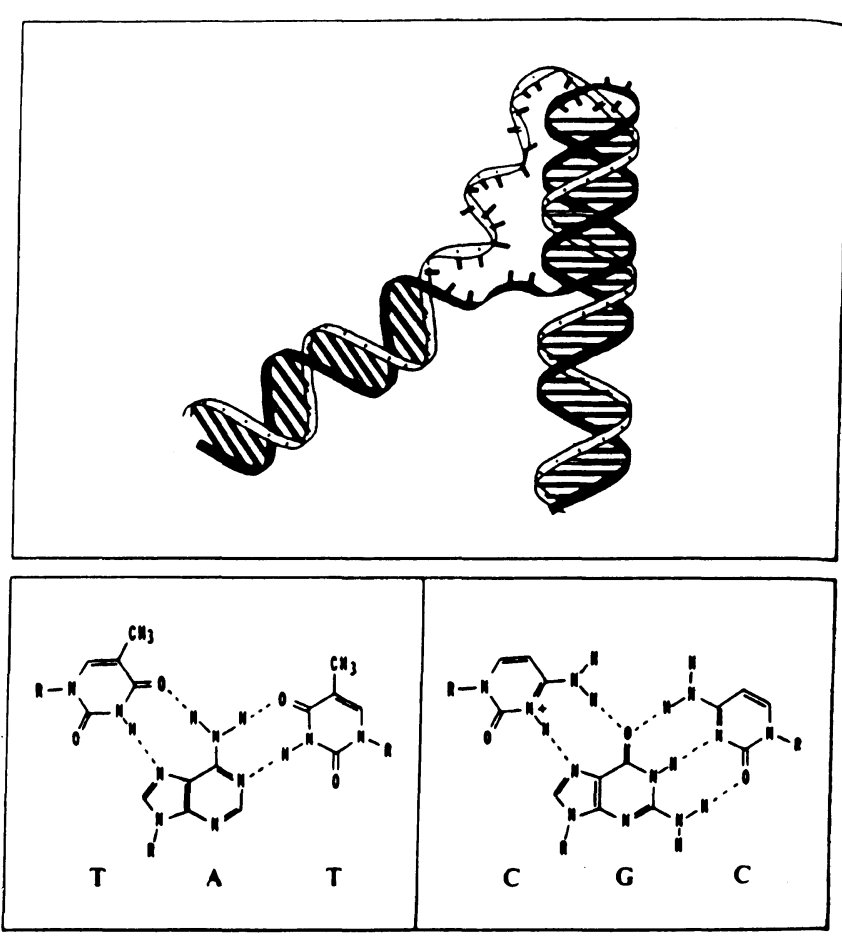

Figure 1. DNA triplex and hydrogen bond formation. 

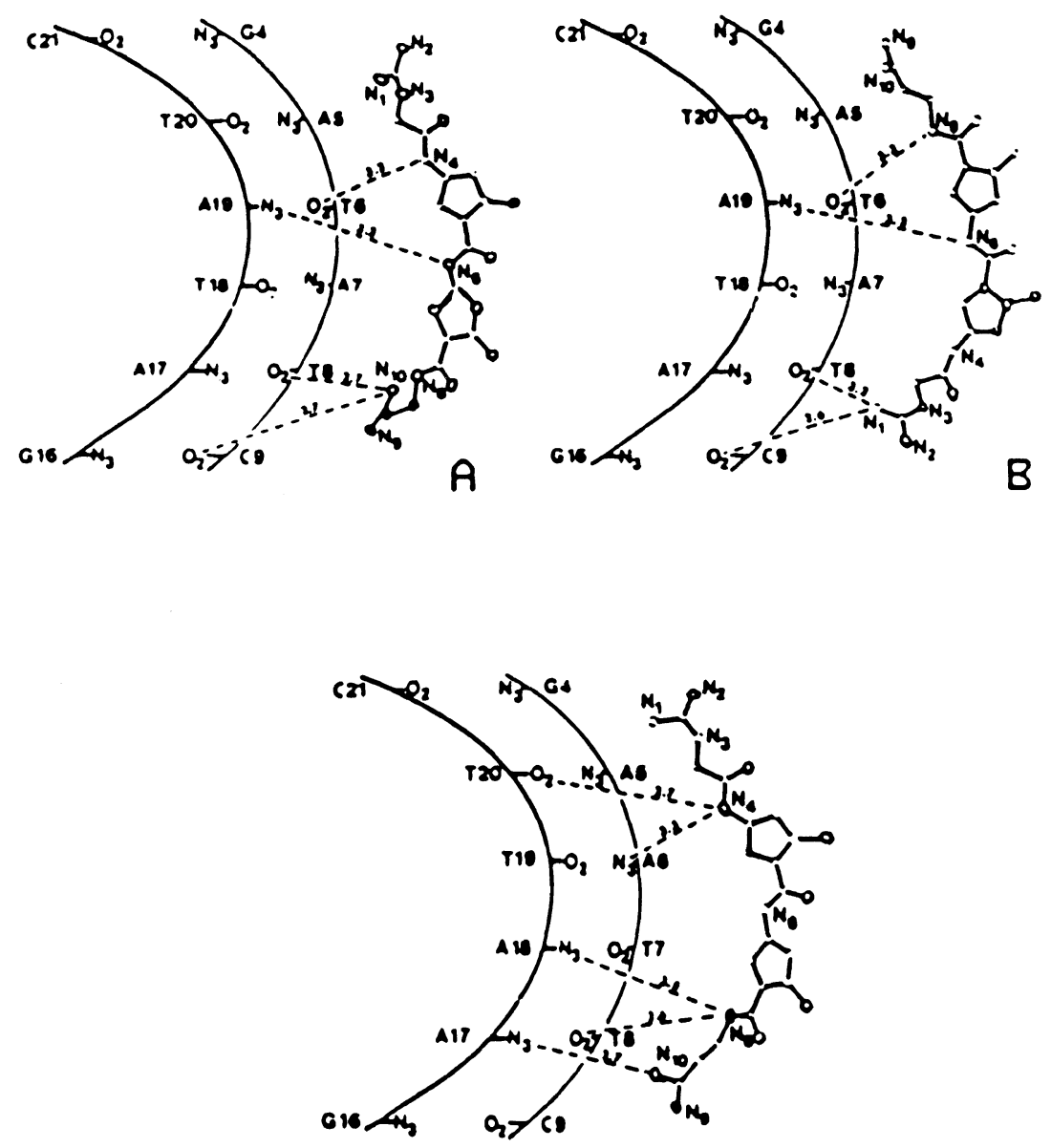

C

Figure 2. Netropsin binding to DNA. A and B, the Netropsin-d(CGCGATATCGCG) complex in two different orientations of Netropsin; C, the Netropsin-d(CGCGAATTCGCG) complex.

strand components. The triplex formation and stability depend upon the salt concentration, and increases with the $\mathrm{Na}^{+}$ions. Similar results were observed by Park et al. ${ }^{10}$ for $\mathrm{dT} \cdot \mathrm{dA} \cdot \mathrm{dT}$ triplex, each strand having 101 bases (202 base pairs). In their studies the influence of netropsin binding in the minor groove of DNA triplex (Figure 2) was characterized by using spectroscopic and calorimetric techniques. They reported that each triplex (Net free and Net bonded) melts in two steps. The first step involves separation of only third strand and the second, melting of DNA duplex and the drug. CD spectra of the third strand 5'-TTTTTCTCTCTCTCT-3' synthesized by us shows that it exists in helical form. This gives negative Cotton effect which is typical of helical conformation. Hence the first step in the transition is the order-order transition whereas the second is the order-disorder one.

Drug binding destabilises the triplex and stabilizes the duplex..$^{10,18,20-23}$ To understand such different roles, we should understand not only binding modes but also energetics of the ligand binding to oligonucleotides. In the present communication an attempt has been made to understand the energetics through phase transition with and without drug binding.

Heat capacity is a very important thermodynamic quantity which characterizes the conformational and dynamical state of a biopolymeric system. Around the transition point, heat capacity can be obtained from the expression for free energy derivable from the transition formalism.

In earlier publications our group has reported orderorder and order-disorder transitions in a variety of systems including polynucleotides and phospholipids. ${ }^{24,25}$ In continuation of this, in the present work, we have made an effort to explain order-order and order-disorder transition in DNA triple helices. Our study is based on the model chosen by Plum et al. ${ }^{9}$ and Park et al. ${ }^{10}$ Their absorbance and heat capacity measurements have been used as experimental data. The Zimm and Bragg theory ${ }^{26}$ for helix-coil transition has been suitably amended to explain order-order and order-disorder transitions in DNA triplexes. The effect of netropsin binding in the minor groove has been explained theoretically by observing the nucleation parameter. We have also tried to explain the heat capacity measurements in the neighborhood of the transition. Lambda point anomalies have been explained by a further modification of the same theory.

\section{THEORY}

The Zimm and Bragg theory ${ }^{26}$ has been modified to study the order-order and order-disorder transitions in a DNA triplex. This theory has been used earlier also for similar transitions in biopolymers, phospholipids, and DNA. ${ }^{24,25,27-29}$ 


\section{Order-Order Transition}

In brief if we assume $\sigma_{1}$ and $\sigma_{2}$ to be nucleation parameters and $s_{1}$ and $s_{2}$ growth parameters in $h_{1}$ and $h_{2}$ where $h_{1}$ and $h_{2}$ are two ordered states. Then, the transition matrix can be written as

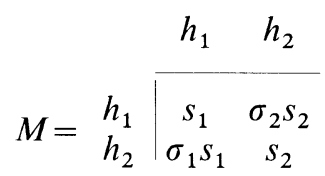

The vector $U$ which gives the contribution of the first segment is

$$
U=\left(\sigma^{\prime} s_{1}, \sigma^{\prime \prime} s_{2}\right)
$$

Where $\sigma^{\prime}$ and $\sigma^{\prime \prime}$ are the parameters giving the interactions of end segments with the solvent when they are in $h_{1}$ and $h_{2}$, respectively. The partition function for an $N$-segment chain is given as

$$
Z=U M^{N-1} V
$$

Where the column vector $V$

$$
V=\left|\begin{array}{l}
1 \\
1
\end{array}\right|
$$

gives the state of the last segment.

Going through the matrix evaluation formalism outlined by Zimm and Bragg, we obtain an expression for the fraction of segments in the form I

$$
f_{1}=\frac{1}{N} \frac{\delta \ln Z}{\delta \ln s_{1}}
$$

solving this we obtain

$f_{1}=\frac{\left[\frac{s_{1}}{\lambda_{1}} \frac{\delta \lambda_{1}}{\delta s_{1}}+B \frac{s_{1}}{\lambda_{2}} \frac{\delta \lambda_{2}}{\delta s_{1}}+\frac{1}{N}\left[\frac{s_{1}}{A_{1}} \frac{\delta A_{1}}{\delta s_{1}}+B \frac{s_{1}}{A_{2}} \frac{\delta A_{2}}{\delta s_{1}}\right]\right]}{(1+B)}$

Here $\lambda_{1}$ and $\lambda_{2}$ are the eigen values of $M$

$$
B=\left[\frac{X_{2}}{X_{1}}\right]^{N}\left[\frac{A_{2}}{A_{1}}\right]
$$

with

$$
\begin{gathered}
X_{1}=s_{1} \lambda_{1}, \quad X_{2}=s_{1} \lambda_{2} \\
A_{1}=\frac{s_{1}+s_{2} x-b \lambda_{2}}{\lambda_{1}-\lambda_{2}}, \quad A_{2}=\frac{s_{1}+s_{2} x-b \lambda_{1}}{\lambda_{2}-\lambda_{1}}, \\
b=\frac{1+x-\sigma_{2}-\sigma_{1} x}{1-\sigma_{1} \sigma_{2}}
\end{gathered}
$$

and

$$
\begin{aligned}
& x=\sigma^{\prime \prime} / \sigma^{\prime} \\
& \frac{s_{1}}{\lambda_{1}} \frac{\delta \lambda_{1}}{\delta s_{1}}=\frac{X_{1}-1}{X_{1}-X_{2}} \quad \frac{s_{1}}{\lambda_{2}} \frac{\delta \lambda_{2}}{\delta s_{1}}=\frac{X_{2}-1}{X_{2}-X_{1}} \\
& \frac{s_{1}}{A_{1}} \frac{\delta A_{1}}{\delta s_{1}} \\
& =\frac{s^{2}(2 \sigma-1+b-x-b \sigma)+s(1+x-b+b \sigma-2 \sigma x)}{\left((1-s)^{2}+4 \sigma x\right)\left(s+x-b X_{2}\right)}
\end{aligned}
$$

$$
\begin{aligned}
& \frac{s_{1}}{A_{2}} \frac{\delta A_{2}}{\delta s_{1}} s^{2}(2 \sigma-1+b-x-b \sigma)+s(1+x-b+b \sigma-2 \sigma x) \\
& \quad=\frac{\left((1-s)^{2}+4 \sigma x\right)\left(s+x-b X_{1}\right)}{}
\end{aligned}
$$

where

$$
\sigma=\sigma_{1} \sigma_{2} \text { and } s=s_{1} / s_{2}
$$

Total absorbance of the nucleotide is given by

$$
\text { Absorbance }=f_{1} A_{1}+\left(1-f_{1}\right) A_{2}
$$

where $A_{1}$ and $A_{2}$ are absorbance in first and second states.

The extension of this for specific heat is straightforward. The specific heat is related to molar enthalpy and entropy change in the transition from states I to II. From well known thermodynamic relations, free energy $F$ is given by

$$
F=-K T \ln Z
$$

and internal energy

$$
U=-T^{2} \frac{\delta}{\delta T}\left[\frac{F}{T}\right]
$$

Differentiating this equation with respect to temperature and using

$$
S=\exp \left[\frac{\Delta H}{R}\left[\frac{1}{T}-\frac{1}{T_{\mathrm{f}}}\right]\right]
$$

where $\Delta H$ is molar change in enthalpy about the transition point and $T_{\mathrm{f}}$ is the transition temperature, we obtain specific heat as

$$
C_{\mathrm{v}}=\delta U / \delta T=N k(\Delta H / R T)^{2}\left(s \delta f_{1} / \delta s\right)
$$

where

$$
\begin{aligned}
\frac{\delta f_{1}}{\delta s}= & -\frac{f_{1}}{(1+B)} \frac{\delta B}{\delta s}+\frac{1}{(1+B)}\left[\frac{\delta}{\delta s}\left[\frac{s_{1}}{\lambda_{1}} \frac{\delta \lambda_{1}}{\delta s_{1}}\right]\right. \\
& +\frac{1}{N} \frac{\delta}{\delta s}\left[\frac{s_{1}}{A_{1}} \frac{\delta A_{1}}{\delta s_{1}}\right]+\frac{\delta B}{\delta s}\left[\frac{s_{1}}{\lambda_{2}} \frac{\delta \lambda_{2}}{\delta s_{1}}\right. \\
& \left.+\frac{1}{N} \frac{s_{1}}{A_{2}} \frac{\delta A_{2}}{\delta s_{1}}\right]+B\left[\frac{\delta}{\delta s}\left[\frac{s_{1}}{\lambda_{2}} \frac{\delta \lambda_{2}}{\delta s_{1}}\right]\right. \\
& \left.\left.+\frac{1}{N} \frac{\delta}{\delta s}\left[\frac{s_{1}}{A_{2}} \frac{\delta A_{2}}{\delta s_{1}}\right]\right]\right]
\end{aligned}
$$

and with

$$
\begin{aligned}
& \frac{\delta B}{\delta s}=N B\left[\frac{1 \delta X_{2}}{X_{2} \delta s}-\frac{1 \delta X_{1}}{X_{1} \delta s}\right]+B\left[\frac{1-b\left(\delta X_{1} / \delta s\right)}{\left(s+x-b X_{1}\right)}\right. \\
& \left.-\frac{1-b\left(\delta X_{2} / \delta s\right)}{\left(s+x-b X_{2}\right)}\right] \\
& \frac{\delta X_{1}}{\delta s}=\frac{1}{2}+\frac{(s-1+2 \sigma)}{2\left[(s-1)^{2}+4 \sigma s\right]^{1 / 2}} \\
& \left.\frac{\delta X_{2}}{\delta s}=\frac{1}{2}-\frac{(s-1+2 \sigma)}{2\left[(s-1)^{2}+4 \sigma s\right]^{1 / 2}}\right\} \\
& \frac{\delta}{\delta s}\left[\frac{s_{1}}{\lambda_{1}} \frac{\delta \lambda_{1}}{\delta s_{1}}\right]=\frac{\sigma(s+1)}{\left((s-1)^{2}+4 \sigma s\right)^{3 / 2}} \\
& \left.\frac{\delta}{\delta s}\left[\frac{s_{1}}{\lambda_{2}} \frac{\delta \lambda_{2}}{\delta s_{1}}\right]=\frac{-\sigma(s+1)}{\left((s-1)^{2}+4 \sigma s\right)^{3 / 2}}\right]
\end{aligned}
$$




$$
\begin{aligned}
\frac{\delta}{\delta s} & {\left[\frac{s_{1}}{A_{1}} \frac{\delta A_{1}}{\delta s_{1}}\right]=\left[\left[(1-s)^{2}+4 \sigma s\right]\left[s+x-b X_{2}\right]\right.} \\
& \times[2 S(2 \sigma-1+b-x-b \sigma)+(1+x-b+b \sigma-2 \sigma x)] \\
& -\left[\left(s^{2}(2 \sigma-1+b-x-b \sigma)+s(1+x-b+b \sigma-2 \sigma x)\right)\right] \\
& \times\left[\left((1-s)^{2}+4 \sigma s\right)\left(1-b \frac{\delta X_{2}}{\delta S}\right)\right. \\
& \left.\left.+\left(s+x-b X_{2}\right)(2 s-2+4 \sigma)\right]\right] /\left[\left((1-s)^{2}\right.\right. \\
& \left.+4 \sigma s)\left(s+x-b X_{2}\right)\right]^{2} \\
\frac{\delta}{\delta s} & {\left[\frac{s_{1}}{A_{2}} \frac{\delta A_{2}}{\delta s_{1}}\right]=\left[\left[(1-s)^{2}+4 \sigma s\right]\left[s+x-b X_{1}\right]\right.} \\
& \times[2 S(2 \sigma-1+b-x-b \sigma)+(1+x-b+b \sigma-2 \sigma x)] \\
& -\left[s^{2}(2 \sigma-1+b-x-b \sigma)+s(1+x-b+b \sigma-2 \sigma x)\right] \\
& \times\left[\left((1-s)^{2}+4 \sigma s\right)\left(1-b \frac{\delta X_{1}}{\delta S}\right)\right. \\
& \left.\left.+\left(s+x-b X_{1}\right)(2 s-2+4 \sigma)\right]\right] /\left[\left((1-s)^{2}\right.\right. \\
& \left.+4 \sigma s)\left(s+x-b X_{1}\right)\right]^{2}
\end{aligned}
$$

\section{Order-Disorder Transition}

To explain the second step high temperature transition the theory of order-disorder ${ }^{29}$ transition has been used. The theory involves writing the Ising matrix in terms of segment pair partition functions which are either in random coil form (denoted by $r$ ) or helical form (denoted by $h$ ). The boundary between the helical and random coil states, i.e., the first $h$ segments in a sequence of $h$ states is denoted by a $k$ state. $f_{\mathrm{r}}, f_{\mathrm{h}}$, and $f_{\mathrm{k}}$ are corresponding segment partition functions of the three states. Taking into account the nearest-neighbor interaction the basic matrix for the problem is

$$
\begin{array}{c|ccc}
\multicolumn{1}{c}{r} & k & h \\
\cline { 2 - 4 } M= & \multicolumn{1}{c}{r} \\
\cline { 2 - 4 } & f_{\mathrm{r}}^{1 / 2} f_{\mathrm{r}}^{1 / 2} & f_{\mathrm{r}}^{1 / 2} f_{\mathrm{k}}^{1 / 2} & 0 \\
k & f_{\mathrm{r}}^{1 / 2} f_{\mathrm{k}}^{1 / 2} & 0 & f_{\mathrm{k}}^{1 / 2} f_{\mathrm{h}}^{1 / 2} \\
h & f_{\mathrm{h}}^{1 / 2} f_{\mathrm{r}}^{1 / 2} & 0 & f_{\mathrm{h}}^{1 / 2} f_{\mathrm{h}}^{1 / 2}
\end{array}
$$

The eigenvalues of $M$ are given by

$$
\begin{aligned}
& \lambda_{1}=\frac{1}{2}\left[\left(f_{\mathrm{r}}+f_{\mathrm{h}}\right)+\left\{\left(f_{\mathrm{r}}-f_{\mathrm{h}}\right)^{2}+4 f_{\mathrm{r}} f_{\mathrm{h}}\right\}^{1 / 2}\right] \\
& \lambda_{2}=\frac{1}{2}\left[\left(f_{\mathrm{r}}+f_{\mathrm{h}}\right)-\left\{\left(f_{\mathrm{r}}-f_{\mathrm{h}}\right)^{2}+4 f_{\mathrm{r}} f_{\mathrm{h}}\right\}^{1 / 2}\right] \\
& \lambda_{3}=0
\end{aligned}
$$

The contribution of the first segment to the partition function is given by

$$
U=\left(f_{\mathrm{r}}^{1 / 2}, 0,0\right)
$$

where the column matrix is

$$
V=\left|\begin{array}{l}
f_{\mathrm{r}}^{1 / 2} \\
f_{\mathrm{k}}^{1 / 2} \\
f_{\mathrm{h}}^{1 / 2}
\end{array}\right|
$$
by

The partition function for a $N$-segment chain is given

$$
Z=U M^{N-1} V
$$

On substituting the values from eq 1, 2, 3, 4, and 5 the partition function becomes

$$
Z=C_{1} \lambda_{1}^{N}+C_{2} \lambda_{2}^{N}
$$

where

$$
C_{1}=\left(\lambda_{1}-f_{\mathrm{h}}\right) /\left(\lambda_{1}-\lambda_{2}\right) \quad \text { and } \quad C_{2}=\left(\lambda_{2}-f_{\mathrm{h}}\right) /\left(\lambda_{2}-\lambda_{1}\right)
$$

The fraction of the segments in the random coil form is given by

$$
Q_{\mathrm{r}}=\frac{1}{N} \frac{\delta \ln Z}{\delta \ln f_{\mathrm{r}}}
$$

Solving above equations we get

$$
Q_{\mathrm{r}}=\frac{1}{2}+\frac{(1-S)(2 A-1)}{2 P}+\frac{(1+S)\{(2 A-1) P-1+S\}}{2 P^{2} N}
$$

where

$$
\begin{aligned}
& P=\left(\lambda_{1}-\lambda_{2}\right) / f_{\mathrm{r}}, \quad S=f_{\mathrm{h}} / f_{\mathrm{r}}, \quad \sigma=f_{\mathrm{k}} / f_{\mathrm{r}} \\
& A=\left[\left(f_{\mathrm{r}}-f_{\mathrm{h}}\right)^{2}+4 f_{\mathrm{k}} f_{\mathrm{r}}\right]^{-1 / 2}
\end{aligned}
$$

Hence

$$
\text { Absorbance }=Q_{\mathrm{r}} A_{\mathrm{r}}+\left(1-Q_{\mathrm{r}}\right) A_{\mathrm{h}}
$$

where $A_{\mathrm{r}}$ and $A_{\mathrm{h}}$ are absorbances in random coil and helical forms respectively.

Again making use of eq 9,10 , and 11 , the heat capacity comes out to be

$$
C_{\mathrm{v}}=\delta U / \delta T=N k(\Delta H / R T)^{2}\left(S \delta Q_{\mathrm{r}} / \delta S\right)
$$

where

$$
\begin{aligned}
& \delta Q_{\mathrm{r}} / \delta S=\left(1 / 2 P^{2}\right)[2 P(1-S) \delta A / \delta S-P(2 A-1) \\
& \quad-(1-S)(2 A-1) \delta P / \delta S] \\
& \quad+\left(1 / 2 P^{3} N\right)[P\{(S+1)\{(2 A-1) \delta P / \delta S \\
& \quad+2 P \delta A / \delta S+1\}+\{(2 A-1) P-1+S\}\} \\
& \quad-\{(2 A-1) P-1+S\} 2(S+1)]
\end{aligned}
$$

with

$$
\begin{aligned}
& \delta A / \delta S=\frac{(S-\sigma)^{N}}{\left(Z / f_{r}^{N}\right)^{2}} \times \frac{\sigma}{P^{3}} \times\left[-2+\frac{N(S-2 \sigma-1)}{S-\sigma}\right] \\
& \delta P / \delta S=(S-1) / P \quad \text { and } \quad \sigma=f_{\mathrm{k}} / f_{\mathrm{r}}
\end{aligned}
$$

\section{RESULTS AND DISCUSSION}

All input parameters, i.e., enthalpy change around the transition point for a given chain length, transition temperature, and nucleation parameters are given in Tables I-III. These experimental data have been taken from the work of Plum et al. ${ }^{9}$ and Park et al. ${ }^{10}$

\section{Order-Order Transition}

As remarked earlier the triplex melting takes place in two steps, involving first the expulsion of the third strand and melting of duplex in the next one. Because of the 
Drug Binding and Transitions in DNA Triple Helices

Table I. Various input parameters for the order-order and order-disorder transitions in DNA triplex Y15 $\cdot \mathrm{U} 21 \cdot \mathrm{Y} 21$

\begin{tabular}{|c|c|c|c|c|c|c|c|c|c|c|c|}
\hline Transition & $T_{\mathrm{m}} / \mathrm{K}$ & $\frac{\Delta H}{\begin{array}{c}\text { kcal mol} \\
\text { base pair }\end{array}}$ & $x$ & $\sigma_{1}$ & $\sigma_{2}$ & $\sigma$ & $N$ & $A_{\mathrm{r}}$ & $A_{\mathrm{h}}$ & $\begin{array}{l}\text { Experimental } \\
\text { half width } \\
(\Delta T)\end{array}$ & $\begin{array}{l}\text { Theoretical } \\
\text { half width } \\
\qquad(\Delta T)\end{array}$ \\
\hline $\begin{array}{l}\mathrm{Y} 15 \cdot \mathrm{U} 21 \cdot \mathrm{Y} 21 \rightarrow \\
\quad \mathrm{Y} 15+\mathrm{U} 21 \cdot \mathrm{Y} 21\end{array}$ & 303.0 & 2.0 & 0.6 & $2.0 \times 10^{-3}$ & $9.0 \times 10^{-4}$ & $1.8 \times 10^{-6}$ & 30 & $0.774 *$ & 0.709 & 5.27 & 5.500 \\
\hline $\begin{array}{l}\mathrm{Y} 15+\mathrm{U} 21 \cdot \mathrm{Y} 21 \rightarrow \\
\mathrm{Y} 15+\mathrm{U} 21+\mathrm{Y} 21\end{array}$ & 337.3 & 6.0 & - & - & - & $3.0 \times 10^{-3}$ & 42 & 0.930 & 0.760 & 3.955 & 3.955 \\
\hline $\mathrm{U} 21 \cdot \mathrm{Y} 21 \rightarrow \mathrm{U} 21+\mathrm{Y} 21$ & 337.3 & 6.0 & - & - & - & $3.0 \times 10^{-3}$ & 42 & 0.742 & 0.552 & 3.555 & 3.555 \\
\hline
\end{tabular}

Note: Y15=5'-TTTTTCTCTCTCTCT-3'; U21 = 5'-GCTAAAAAGAGAGAGAGATCG-3'; Y21 = 3'-CGATTTTTCTCTCTCTCTAGC-5'; $A_{\mathrm{r}}=$ absorbance in random state; $A_{\mathrm{h}},{ }^{*}=$ absorbance in the helical state.

Table II. Various input parameters for the order-order and order-disorder transitions in DAN triplex dT $\cdot \mathrm{dA} \cdot \mathrm{dT}$

\begin{tabular}{|c|c|c|c|c|c|c|c|c|c|c|c|}
\hline Transition & $T_{\mathrm{m}} / \mathrm{K}$ & 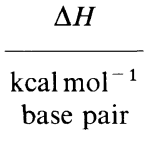 & $x$ & $\sigma_{1}$ & $\sigma_{2}$ & $\sigma$ & $N$ & $A_{\mathrm{r}}$ & $A_{\mathrm{h}}{ }^{\circ}$ & $\begin{array}{l}\text { Experimental } \\
\text { half width } \\
(\Delta T)\end{array}$ & $\begin{array}{l}\text { Theoretical } \\
\text { half width } \\
\qquad(\Delta T)\end{array}$ \\
\hline $\mathrm{dT} \cdot \mathrm{dA} \cdot \mathrm{dT} \rightarrow \mathrm{dT} \cdot \mathrm{dA}+\mathrm{dT}$ & 342.68 & 3.70 & 1.2 & $1.0 \times 10^{-4}$ & $1.0 \times 10^{-2}$ & $1.0 \times 10^{-6}$ & 202 & $1.16^{*}$ & 0.97 & 0.85 & 0.88 \\
\hline $\begin{array}{l}\mathrm{dT} \cdot \mathrm{dA}+\mathrm{dT} \rightarrow \\
\mathrm{dT}+\mathrm{dA}+\mathrm{dT}\end{array}$ & 356.00 & 10.0 & - & - & - & $4.0 \times 10^{-4}$ & 76 & 1.45 & 1.16 & 0.80 & 0.80 \\
\hline $\mathrm{dT} \cdot \mathrm{dA} \rightarrow \mathrm{dT}+\mathrm{dA}$ & 355.70 & 10.0 & - & - & - & $4.0 \times 10^{-4}$ & 76 & 1.254 & 0.91 & 0.83 & 0.83 \\
\hline
\end{tabular}

Note: $A_{\mathrm{r}}=$ absorbance in random state; $A_{\mathrm{h}},{ }^{*}=$ absorbance in the helical state.

Table III. Various input parameters for the order-order and order-disorder transition in DNA triplex $\mathrm{dT} \cdot \mathrm{dA} \cdot \mathrm{dT}$ (netropsin bonded)

\begin{tabular}{|c|c|c|c|c|c|c|c|c|c|c|c|}
\hline Transition & $T_{\mathrm{m}} / \mathrm{K}$ & 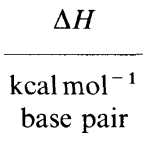 & $x$ & $\sigma_{1}$ & $\sigma_{2}$ & $\sigma$ & $N$ & $A_{\mathrm{r}}$ & $A_{\mathrm{h}}$ & $\begin{array}{l}\text { Experimental } \\
\text { half width } \\
(\Delta T)\end{array}$ & $\begin{array}{l}\text { Theoretical } \\
\text { half width } \\
\qquad(\Delta T)\end{array}$ \\
\hline $\begin{array}{l}\mathrm{dT} \cdot \mathrm{dA} \cdot \mathrm{dT}(\mathrm{NET}) \rightarrow \\
\mathrm{dT} \cdot \mathrm{dA}(\mathrm{NET})+\mathrm{dT}\end{array}$ & 294.9 & 4.6 & 1.2 & 0.35 & $1.0 \times 10^{-4}$ & $1.0 \times 10^{-5}$ & 118 & $0.624^{*}$ & 0.492 & 1.56 & 1.56 \\
\hline $\begin{array}{l}\mathrm{dT} \cdot \mathrm{dA}(\mathrm{NET})+\mathrm{dT} \rightarrow \\
\quad \mathrm{dT}+\mathrm{dA}+\mathrm{dT}+\mathrm{NET}\end{array}$ & 373.0 & 13.9 & - & - & - & $3.5 \times 10^{-3}$ & 30 & 0.89 & 0.63 & 1.25 & 1.25 \\
\hline $\begin{array}{l}\mathrm{dT} \cdot \mathrm{dA}(\mathrm{NET}) \rightarrow \\
\mathrm{dT}+\mathrm{dA}+\mathrm{NET}\end{array}$ & 371.2 & 13.9 & - & - & - & $3.5 \times 10^{-3}$ & 30 & 0.75 & 0.36 & 2.04 & 2.04 \\
\hline
\end{tabular}

Note: $A_{\mathrm{r}}=$ absorbance in random state; $A_{\mathrm{h}},{ }^{*}=$ absorbance in the helical state.

mirror symmetry, the thermally expelled strand is helical in nature and hence the first step transition is an orderorder transition. However, duplex melting results in the transition of the two strands to disordered states. Thus, the second step melting is an order-disorder state transition. The various transition parameters which provide the best fit to the experimental data are given in Tables I, II, and III. The deviation of the end effect parameter from unity (for an infinite chain) is also shown, and is larger for smaller interacting lengths and vice versa. In other words, a finite system has a memory which fades out with increasing length. These end effect parameters play partly the role of scaling factors and may have their origin not only in the finiteness of the chains but also in the nature of interactions and experimental measurements. The value of $N$ depends upon the number of interacting base pairs. Our calculations correspond to $N=30,202$, and 118 for $\mathrm{Y} 21 \mathrm{U} 21 \mathrm{Y} 15 \rightarrow \mathrm{Y} 21 \mathrm{U} 21+\mathrm{Y} 15$, $\mathrm{dT} \cdot \mathrm{dA} \cdot \mathrm{dT} \rightarrow \mathrm{dT} \cdot \mathrm{dA}+\mathrm{dT}$ and $\mathrm{dT} \cdot \mathrm{dA} \cdot \mathrm{dT}(\mathrm{Net}) \rightarrow \mathrm{dT} \cdot$ $\mathrm{dA}(\mathrm{Net})+\mathrm{dT}$ respectively. The theoretical transition curves have been obtained by using eq 7 and 8 . These curves are shown in Figures 3, 5, and 7. It is clear from the Figures that the order-order transition of $d \mathrm{~T} \cdot \mathrm{dA} \cdot \mathrm{dT}$ is sharper than that of the order-order transition of Y21U21Y15 which is due to stronger cooperativity arising from longer chain length of $\mathrm{dT} \cdot \mathrm{dA} \cdot \mathrm{dT}$. This is also evident from the smaller value of sigma and larger change in the enthalpy during the transition $\mathrm{dT} \cdot \mathrm{dA}$. $\mathrm{dT} \rightarrow \mathrm{dT} \cdot \mathrm{dA}+\mathrm{dT}$. The sharpness of the transition decreases ( $\sigma$ increases) when netropsin binds to its minor groove. It is consistent with the fact that netropsin destabilizes the triplex when bound in its minor groove. This observation is also supported by the fact that when netropsin molecule binds itself in the narrow minor groove of the double helix, it does so by a combination of interactions including hydrogen bonds, ionic charge interactions and Vander Waals interactions. The fact that ${ }^{10}$ during the melting of netropsin bonded triplex only the third strand gets separated and netropsin remains bound to the Watson Crick duplex shows that netropsin when bonded to the triplex stabilizes the Watson-Crick pair in it whereas the Hoogsteen pair gets destabilized. Basically netropsin binds itself to the duplex. Structural details concerning the nature of binding are given by Coll et al., ${ }^{21}$ Tabernero et al., ${ }^{22}$ and recently by Nunn et al. ${ }^{23}$ Netropsin makes bifurcated hydrogen 
bonds only with the Watson-Crick duplex. ${ }^{21,22}$ Destabilization of the triplex because of netropsin binding possibly occurs due to repulsion between nonbonded atoms and due to weakening of the hydrogen bonds arising from the redistribution of charge in the benzene rings. The third strand is strained due to change in base pair propellor angles. ${ }^{21-23}$ The ionic charge and Vander Waals attractions do not adequately compensate for this weakening. Thus, netropsin binding in the minor groove reduces the cooperative unit from 101.3 to $59.2 .{ }^{10}$ All the theoretical transition curves are in very good agreement with the observed experimental data of Plum et $a l{ }^{9}$ The nucleation parameters which provide the best fit for the transition profiles also successfuly generate the $\lambda$-point heat capacity anomaly.

\section{Order-Disorder Transition}

The second sharp transition at the higher temperature which involves the thermal disruption of the duplex to form two single strands has been explained by applying the theory of order-disorder transition. This is so because all experimental evidence indicates that both the strands on separation go into random coil states. There is no mirror symmetry in the strands. Theoretical transition and heat capacity curves for these transitions have been calculated by using eq 27, 29, and 30 . They are shown in Figures 3-8. $N$ is equal to 42,76 , and 30 for $\mathrm{U} 21 \cdot \mathrm{Y} 21$, netropsin free $\mathrm{dT} \cdot \mathrm{dA}$ and netropsin bonded $\mathrm{dT} \cdot \mathrm{dA}$ duplexes respectively. Various transition parameters, i.e., enthalpy change around the transition point for a given chain length, transition temperature, and nucleation parameter for the high temperature transition are in an excellent agreement with the various parameters of the duplex $\rightarrow$ one + one transition. The absorbance during triplex $\rightarrow$ duplex + one is lower as compared to the absorbance during duplex + one $\rightarrow$ one + one + one transition. The absorbance is further reduced during duplex to one + one transition. This difference may be due to the contribution to absorbance due to the third strand and conformational change during transition. From the Figures 3, 5, and 7 it is also clear that the second transition is sharper than the transition from triplex to duplex + one. This is obviously due to the larger value of enthalpy change during transition. Further it is again noted that the high temperature transition of $\mathrm{dT} \cdot \mathrm{dA} \cdot \mathrm{dT}$ is sharper than the corresponding transition of $\mathrm{Y} 15 \cdot \mathrm{U} 21 \cdot \mathrm{Y} 21$ and the sharpness of transition decreases as the netropsin binds into the minor groove of $\mathrm{dT} \cdot \mathrm{dA} \cdot \mathrm{dT}$ triplex. The values of sigma are consistent with this. They are $3 \times 10^{-3}, 4 \times 10^{-4}$, and $3.5 \times 10^{-3}$ in the case of $\mathrm{U} 21 \cdot \mathrm{Y} 21 \rightarrow \mathrm{U} 21+\mathrm{Y} 21, \mathrm{dT} \cdot \mathrm{dA} \rightarrow \mathrm{dT}+\mathrm{dA}$, and $\mathrm{dT} \cdot$ $\mathrm{dA}(\mathrm{Net}) \rightarrow \mathrm{dA}+\mathrm{dT}+\mathrm{Net}$ transitions, respectively. Enthalpy changes during these transitions are 6.0, 10.0, and $13.9 \mathrm{kcal} \mathrm{mol}^{-1}$ base pairs respectively. Netropsin binding to the DNA duplex thermally stabilizes the duplex as a result of which netropsin bonded duplex melts at a higher temperature in comparison to the netropsin free duplex.

\section{Heat Capacity}

Heat capacity is an important thermodynamic quantity which characterizes the conformational and dynamical states of a macromolecular system. Heat capacity measurements for $\mathrm{Y} 15 \cdot \mathrm{U} 21 \cdot \mathrm{Y} 21, \mathrm{dT} \cdot \mathrm{dA} \cdot \mathrm{dT}$, and $\mathrm{dT} \cdot \mathrm{dA} \cdot \mathrm{dT}(\mathrm{Net})$ are reported by Plum et al. ${ }^{9}$ and Park et al. ${ }^{10}$ Heat capacity is the second derivative of the Gibb's free energy and has been calculated by using the eq 12 and 30 . The heat capacity curves with $\lambda$ point

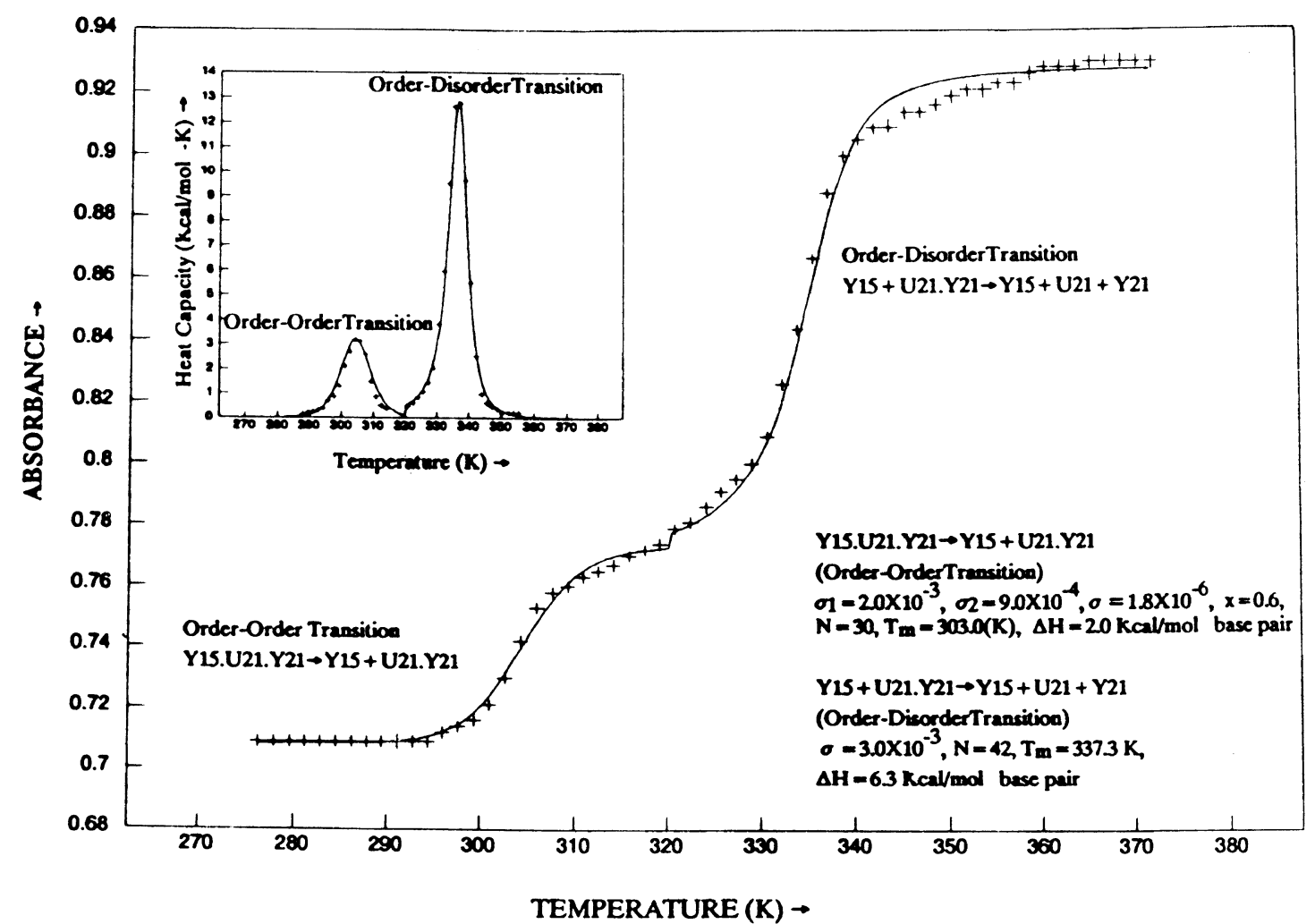

Figure 3. Transition profiles and heat capacity variation (shown in insets) for $\mathrm{Y} 15 \cdot \mathrm{U} 21 \cdot \mathrm{Y} 21 \rightarrow \mathrm{Y} 15+\mathrm{U} 21 \cdot \mathrm{Y} 21 \rightarrow \mathrm{Y} 15+\mathrm{U} 21+\mathrm{Y} 21$ transitions. Calculated values $(-)$; experimental values $(++++++)$. 


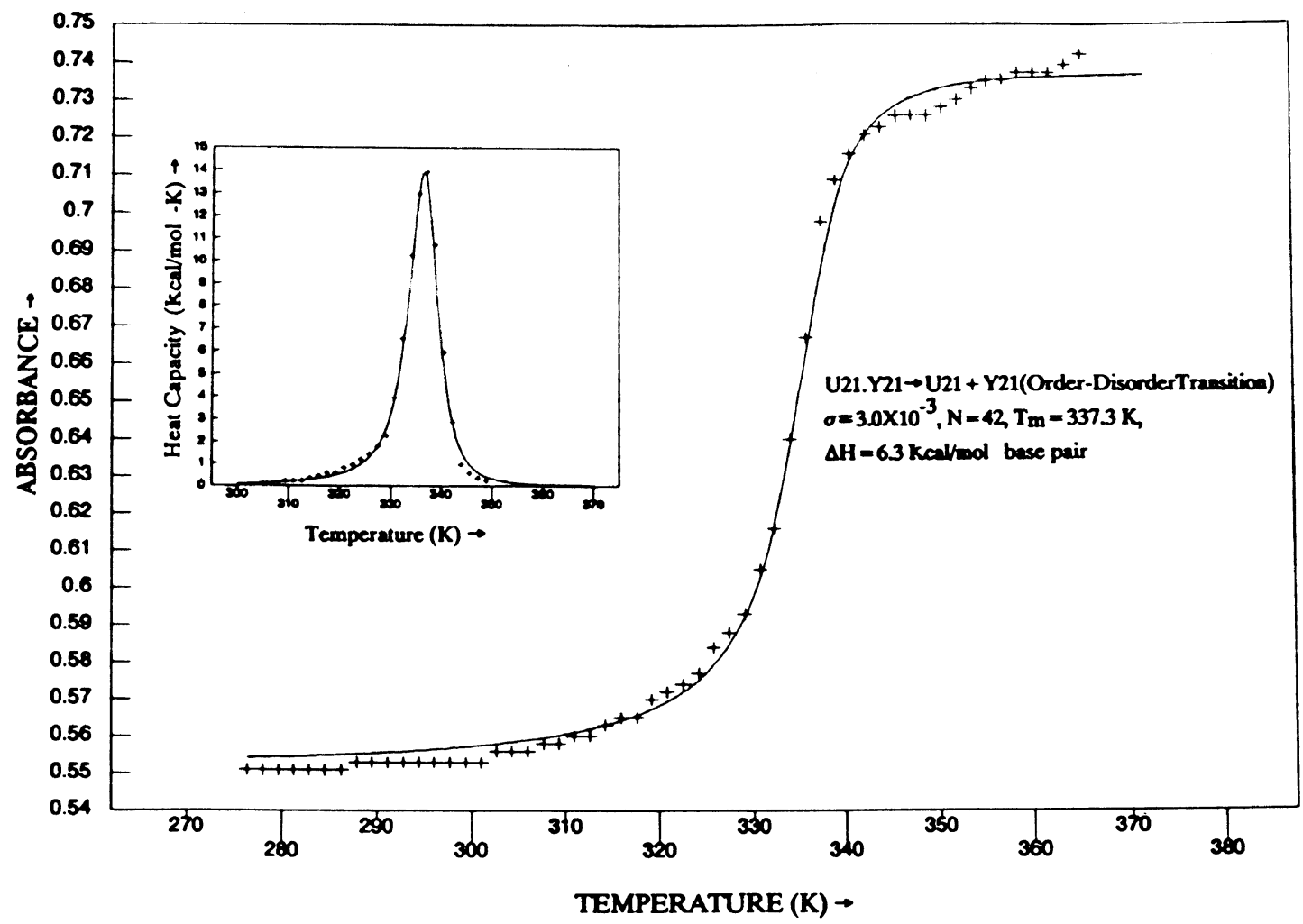

Figure 4. Transition profile and heat capacity variation (shown in inset) for $\mathrm{U} 21 \cdot \mathrm{Y} 21 \rightarrow \mathrm{U} 21 \cdot \mathrm{Y} 21$ transition. Calculated values ( - ); experimental values $(++++++)$.

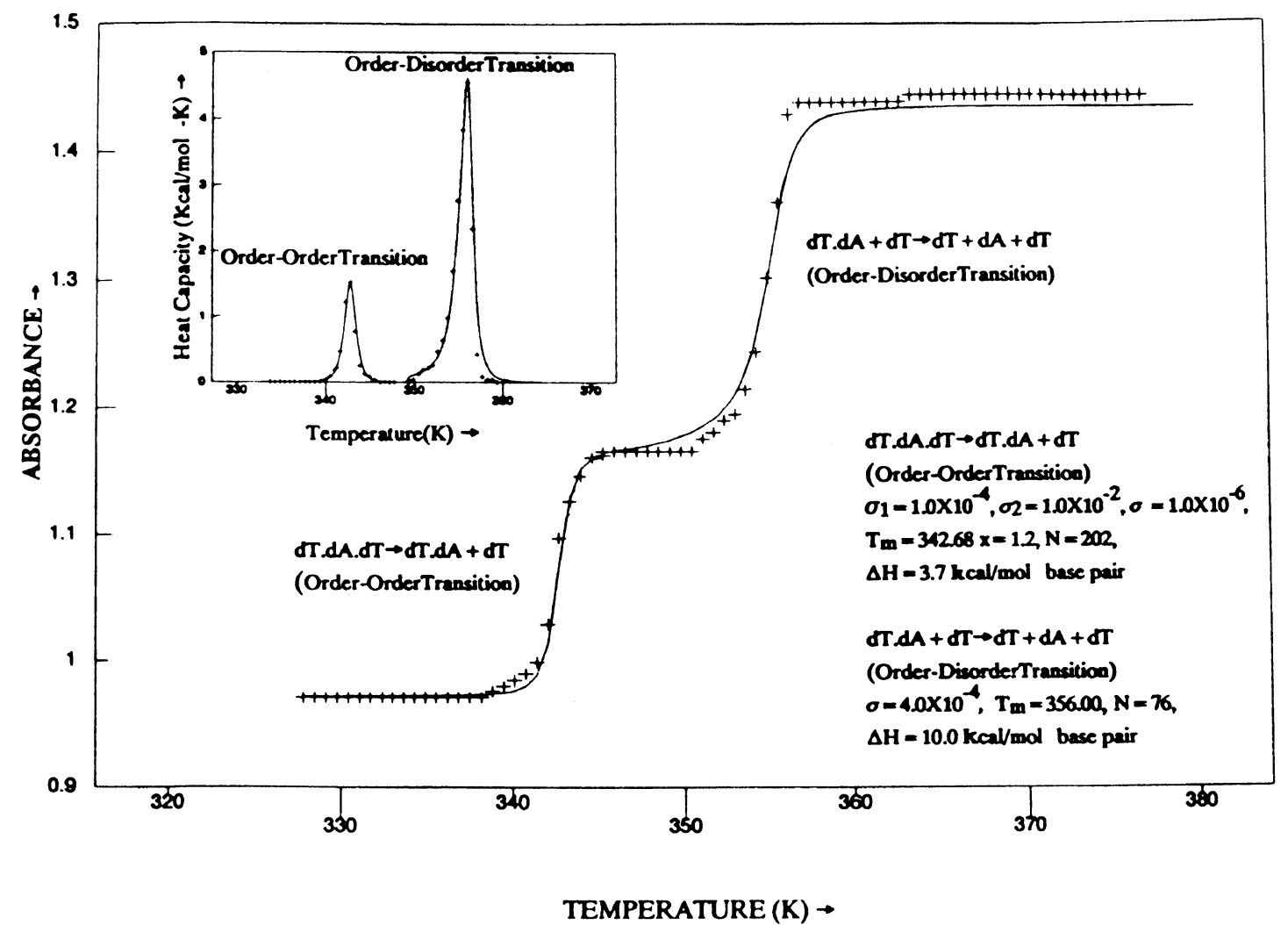

Figure 5. Transition profiles and heat capacity variation (shown in insets) for $d T \cdot d A \cdot d T \rightarrow d T+d A \cdot d T \rightarrow d T+d A+d T$ transitions. Calculated values (-); experimental values $(++++++)$.

anomaly for various transitions are shown as insets in Figures 3-8 along with the transition profiles. Wherever available the experimental data points are also shown. The theoretically obtained heat capacity profiles agreed with the experimentally reported ones and could be brought almost into coincidence with the use of scaling factors. These factors take into account constant error in experimental measurements which cause constant shift 


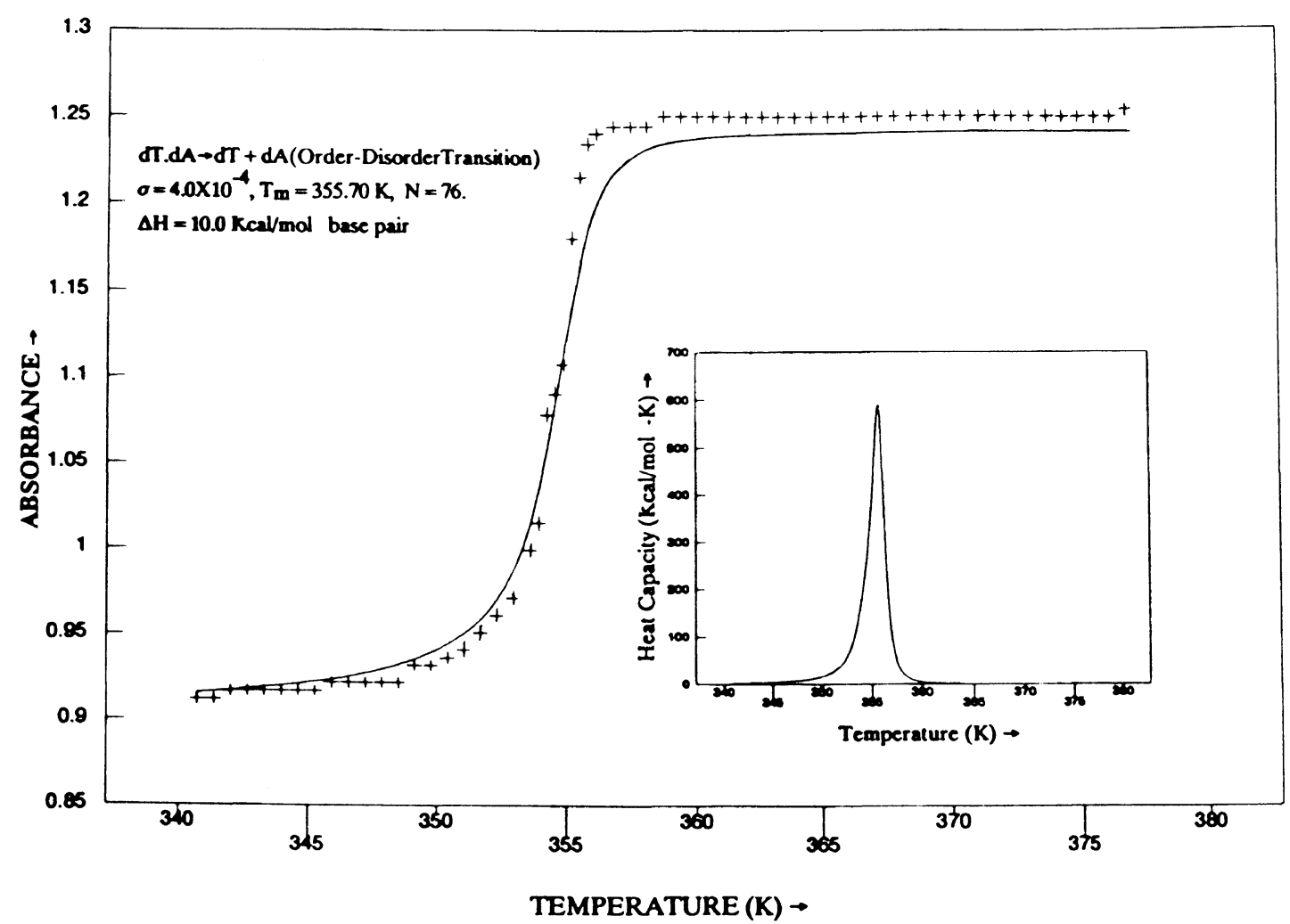

Figure 6. Transition profile and heat capacity variation (shown in inset) for $\mathrm{dT} \cdot \mathrm{dA} \rightarrow \mathrm{dT}+\mathrm{dA}$ transition. Calculated values $(\longrightarrow)$; experimental values $(+++++)$.

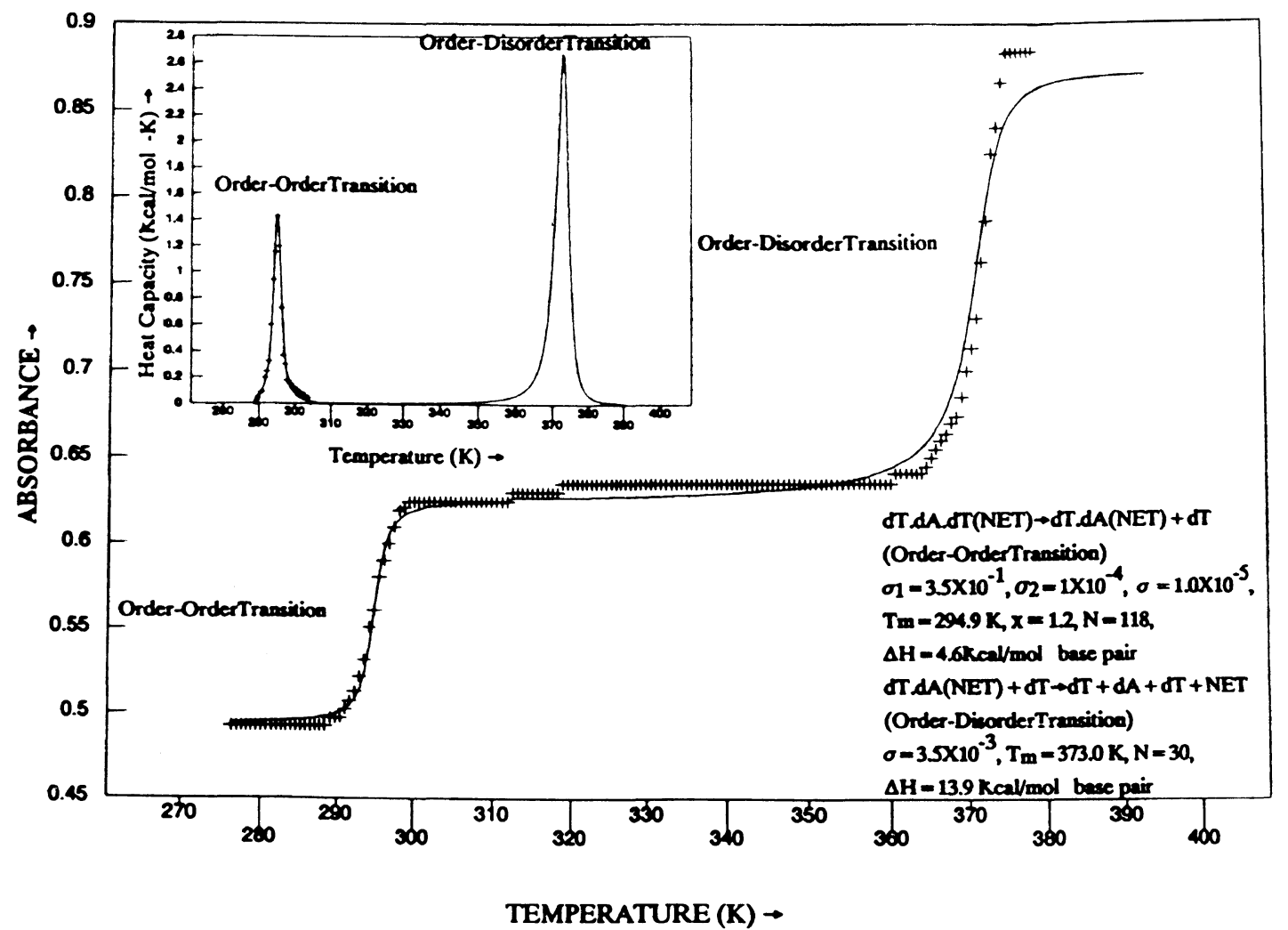

Figure 7. Transition profiles and heat capacity variation (shown in insets) for $d T \cdot d A \cdot d T(N e t) \rightarrow d T+d A \cdot d T(N e t) \rightarrow d T+d A+d T+N e t$ transitions. Calculated values $(-)$; experimental values $(++++++)$.

of the experimental curves. The same can be said of theoretical modelling. The sharpness of the transition can be characterized by the half widths of the heat capacity curves given in Tables I-III. The experimental half widths are in good agreement. The half widths of the order-order and order-disorder transitions in dT. $\mathrm{dA} \cdot \mathrm{dT}$ are smaller than the corresponding transitions in $\mathrm{dT} \cdot \mathrm{dA} \cdot \mathrm{dT}(\mathrm{Net})$. This is a result of the netropsin binding 


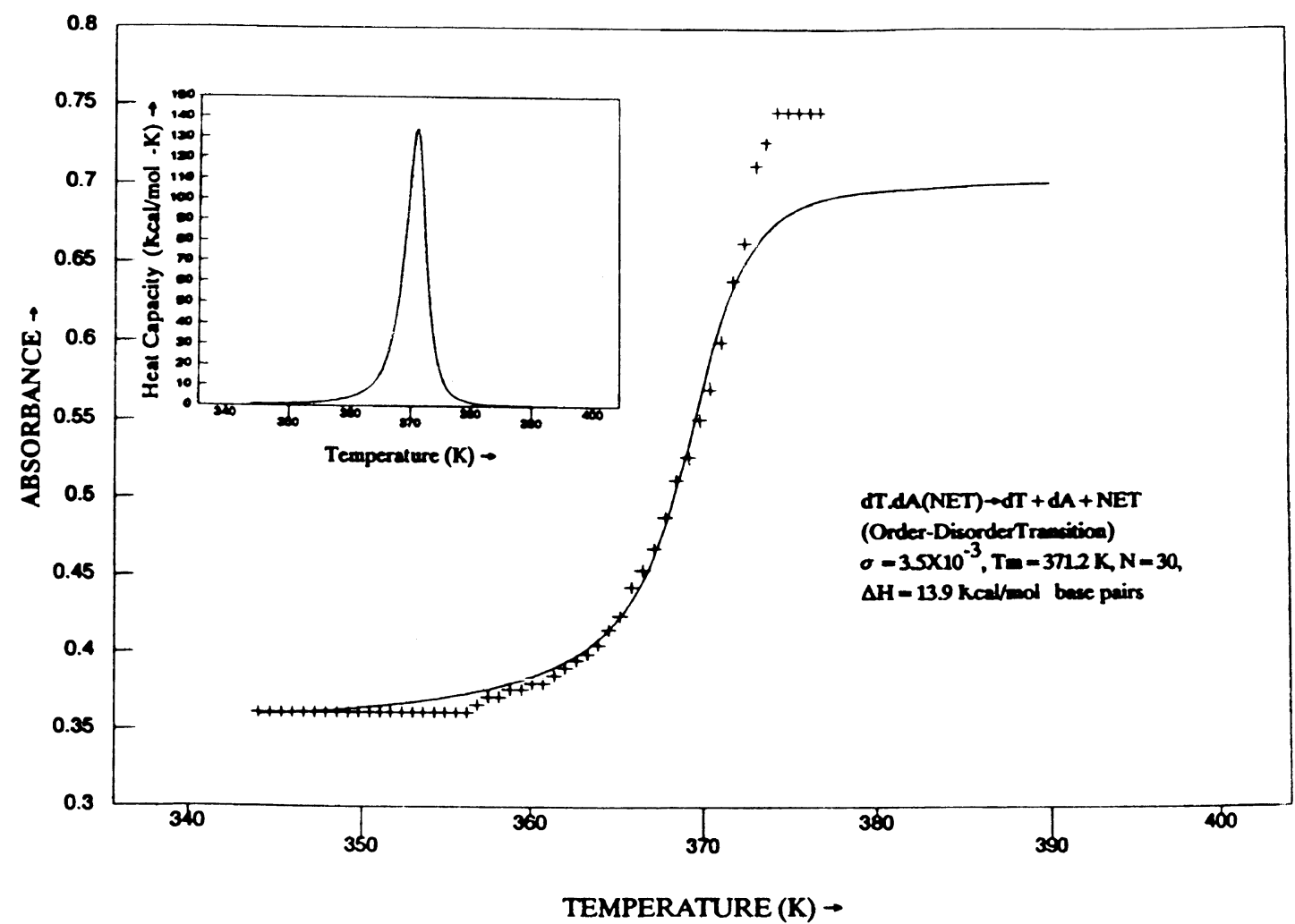

Figure 8. Transition profile and heat capacity variation (shown in inset) for $d A \cdot d T(N e t) \rightarrow d A+d T+N e t$ transitions. Calculated values $(\longrightarrow)$; experimental values $(+++++)$.

which contributes to the stability of the duplex. In case of order-order transition the lambda curves also have a certain degree of asymmetry in the tail end regions. This kind of asymmetry does not appear in the theoretical curves of order-disorder transition due to order-disorder transition depending on a single $\sigma$ value whereas the order-order transition is generated by two $\sigma$ values namely $\sigma_{1}$ and $\sigma_{2}$. Hence the profile of the left and right halves of the heat capacity curves is affected by both values of $\sigma$. In the case of the triplex $\rightarrow$ duplex + one the heat capacity has a smaller value compared to the transition from duplex to one + one and duplex + one $\rightarrow$ one + one + one. This is due to the larger enthalpy change in the last two transitions involving helix to coil states. Since the coiled state is not a uniquely defined state, the heat capacity differences could also arise due to the presence of short helical segments present in the random coil states.

\section{CONCLUSIONS}

The phase transition in the Net-free and Net-bound triple helical DNA can be successfully interpreted within the theoretical framework of Zimm and Bragg $^{26}$ using only two fitting parameters, i.e., the nucleation parameter and end-effect parameter (scaling factor being very close to one). Total theoretical interpretation has been attempted in all parts of the transition profile. Half widths are indicators of the extent of cooperativity. Very good agreement is obtained between the half widths of experimental and theoretical transition profiles.

Acknowledgments. Financial assistance to V.D.G. and S.S. (Research Associate) from the Council of
Scientific and Industrial Research, New Delhi under the Emeritus Scientist Scheme and Research Associateship and to P.T. from the Department of Science and Technology, New Delhi under the Y.S.S. is gratefully acknowledged.

\section{REFERENCES}

1. S. Arnott and P. J. Bond, Nature, 244, 99 (1973).

2. G. Manzini, L. E. Xodo, and D. Gasparotto, J. Mol. Biol., 213, 833 (1990)

3. S. Arnott and E. Selsing, J. Mol. Biol., 88, 509 (1974).

4. D. S. Pilch, C. Levenson, and R. H. Shafer, Proc. Natl. Acad. Sci. U.S.A., 87, 1942 (1990).

5. S. L. Broitman, D. D. Im, and J. R. Fresco, Proc. Natl. Acad. Sci. U.S.A., 83, 5120 (1987).

6. G. C. Silver, J. S. Sun, C. H. Nguyen, A. S. Boutorine, E. Bisagni, and C. Helene, J. Am. Chem. Soc., 119, 263 (1997).

7. G. Felsenfeld, D. R. Davies, and A. Rich, J. Am. Chem. Soc., 79, 2023 (1957).

8. S. A. Strobel, H. E. Moser, and P. B. Dervan, J. Am. Chem. Soc., 110, 7927 (1988).

9. G. E. Plum, Y. W. Park, S. F. Singleton, P. B. Dervan, and K. J. Breslauer, Proc. Natl. Acad. Sci. U.S.A., 87, 9436 (1990).

10. Y. W. Park and K. J. Breslauer, Proc. Natl. Acad. Sci. U.S.A., 89, 6653 (1992)

11. J. Volker, S. E. Osborne, G. D. Glick, and K. J. Breslauer, Biochemistry, 36, 756 (1997).

12. R. W. Robert and D. M. Crothers, Proc. Natl. Acad. Sci. U.S.A., 88, 9397 (1991).

13. K. J. Breslauer, J. M. Sturteevant, and I. T. Jr., J. Mol. Biol., 99, 549 (1975).

14. M. Rougee, B. Faucon, J. L. Mergny, F. Barcelo, C. Giovannangeli, T. Garestier, and C. Helen, Biochemistry, 31, 9269 (1992).

15. J. Volker and H. H. Klump, Biochemistry, 33, 13502 (1994).

16. L. C. Griffin and P. B. Dravan, Science, 245, 967 (1989).

17. H. Htun and J. E. Dahlberg, Science, 243, 1517 (1989).

18. M. Durand and J. C. Maurizot, Biochemistry, 35, 9133 (1996). 
19. R. W. Robert and D. M. Crothers, Science, 258, 1463 (1992).

20. M. Durand, N. T. Thuong, and J. C. Maurizot, J. Biol. Chem., 267, 24394 (1992).

21. M. Coll, J. Aymami, G. A. Vander Marel, J. H. V. Boom, A. Rich, and A. H. J. Wang, Biochemistry, 28, 310 (1989).

22. L. Tabernero, N. Verdaguer, M. Coll, I. Fita, G. A. Vander Marel, and J. H. V. Boom, Biochemistry, 32, 8403 (1993).

23. C. M. Nunn, E. Garman, and S. Neidle, Biochemistry, 36, 4792 (1997).

24. P. Tandon, V. D. Gupta, C. Mehrotra, and D. C. Gupta, J.
Macromol. Sci.-Phys., 31, 439 (1992).

25. P. Tandon, V. D. Gupta, C. Mehrotra, and D. C. Gupta, J. Macromol. Sci.-Phys., 32, 205 (1992).

26. B. H. Zimm and J. K. Bragg, J. Chem. Phys., 31, 526 (1959).

27. R. C. Agnihotri, C. Mehrotra, V. D. Gupta, and V. Srivastava, Pramana, 17, 361 (1981).

28. V. D. Gupta and A. K. Gupta, J. Phys. Soc. Jpn., 20, 841 (1965).

29. U. Kapoor, V. D. Gupta, and C. Mehrotra, J. Phys. A: Gen. Phys., 4, 549 (1971) 